LBL-33294

UC-410

LSGN-128

\title{
MAGNET POWER SUPPLY SYSTEM FOR THE ALS STORAGE RING AND BOOSTER
}

L.T. JACKSON, K. LUCHINI, and I. LUTZ

\author{
ACCELERATOR and FUSION RESEARCH DIVISION \\ Lawrence Berkeley Laboratory \\ University of California \\ Berkeley, CA 94720
}

MAY 1993

This work was supportsis by the Director, Office of Energy Research, Office of Basic Energy Sciences, Materials Sciences Division, of the U.S. Department of Energy under Contract No. DE-AC03-76SF00098. 


\title{
Magnet Power Supply System for the ALS Storage Ring and Booster*
}

\author{
L. Terry Jackson, Ken Luchini, and Ivan Lutz \\ Lawrence Berkeley Laboratory, University of California \\ 1 Cyclotron Road, Berkeley, CA 94720 USA
}

\section{Abstract}

The Magnet Power Supply System is described by specification, design, hardware, and operating experience. A unique system for the one $\mathrm{Hz}, 1.5 \mathrm{GeV}$ Booster, where the wideband QF and QD power supplies track the dipole current to within $0.1 \%$ at injection will be detailed. AC distribution system considerations related to inverting the stored energy of the booster magnet back into power grid will be discussed. The rational for linear correctors and individual quad supplies (225 total units) will be placed within the context of the storage-ring requirements.

\section{LINAC TO BOOSTER MAGNET P.S.}

The power supplies for this transport line are EMI Power Supplies for the dipole magnets, Kepco power supplies for the quadrupole magnets and Techron power amplifiers for the vertical,horizontal correctur magnets. The attempt in this transport line system was to use commercially available equipment in totality. . The EMI Power Supplies were bought with amplifiers added so that the input and output interface was zero to $10 \mathrm{~V}$ in both cases to aliow interfacing with the Intelligent Local Controllers (ILC) used throughout the ALS system. The Kepco supplies were modified at LBL by adding components to the interface plug located on the back of the Kepco to make a high-gain close-loop current control system. The utilization of the interface plug-in allowed use of the two amplifiers internally mounted in the Kepco to again provide input and output reference and feedback signals at 0 to $10 \mathrm{~V}$. The. Techron Supplies could not be internally modified to allow input and output interfacing at 0 to $10 \mathrm{~V}$. An attempt was made to do this internally by changirig the gain of the amplifiers but internal wiring practices cause oscillations to occur when the gains were raised on these amplifiers so the decision was made to fabricate an interface chassis, each to interface to four of the Techrons on the LTB line. There is a divider to lower the input reference from 10 volts to the 0.6 volts maximum required by the Techron and then an amplifier to take the $0.6 \mathrm{~V}$ max. outputs current monitor signal up to the $10 \mathrm{~V}$ required by the ILCs. The basic level of operation of this witule system was $\pm 0.1 \%$ of the maximum current, and with the components as described the system has proven to work satisfactorily.

\section{BOOSTER POWER SUPPLY SYSTEM}

As was described in the 1989 paper[1] on the plans for this system, the booster power-supply system operates at $1 \mathrm{~Hz}$ and therefore it was not necessary to use a resonant system (using a White circuit for instance) as has been done on systems that operate at higher frequencies (typically $10 \mathrm{~Hz}$ and above). The supply for the ALS Booster Dipole string of magnets runs as an open loop 12 pulse supply with a 0 or $10 \mathrm{~V}$ control signal

*This work was supported by the Director, Office of Energy Research, Office of Basic Energy Sciences, Materials Sciences Division, of the U.S.DOE, under Contract No. DE-AC03-76SF00098. and goes into full rectification for ramping up the current, and then into almost full inversion for completion of the cycle and returning the current to zero. This approach was chosen for minimum cost knowing that the current would vary from pulse to pulse as the line voltage varies, and this it does.

The quadrupole (QF and QD families of magnets) supplies are closed-loop high-performance systems that track the dipole current and make it possible to have the large 1 MVA dipolesupply running without any closed loop operation. This approach has worked very satisfactorily both for tracking and perturbations of the dipole current. The equipment from the manufacturer, with some modifications, has proven eminently satisfactory for our operation. The crucial requirement on the ramp operation is that the quadrupole supplies are tracking the dipole current at injection, which is approximately $1 / 30$ of the maximum energy (and 10 msecs from start of ramp), so they must have wide bandwidth to be able to catch up and have the performance necessary at the time of injection. The sextupole and corrector supplies are small units and they also track the dipole current as their reference.

The dipole reference current signal $(0$ to $10 \mathrm{~V})$ is distributed to the quadrupole sextupole, and corrector circuits through an interface circisit that allows correction to be made to the basic booster current signal as a function of energy level for each power supply. 'Theic are iwo parts to this interface. One is the digital-multiplier circuit which allows each circuit to have its own level of reference as a percentage of the 0 to 10 $\mathrm{V}$ peak signal from the dipole transductor. The other element of the circuit is a summer which is controlled from a look-up table in the ILC which gets timing information from energy markers generated by the timing system (approximately 5 msecs repetition rate at injection). The small correction $(0.1 \%)$ necessary on the basic dipole signal is particularly important at injection and extraction energies. Hysteresis effects are the main sources of error between the dipole signal and the required quadruf jle signal at injection. Saturation effects require small correction at extraction energy levels. All the corrections for the supplies are done by the operators in the control room through the ILC system. The concept and execution of this approach have worked out well and the booster operation is today basically push-button operation using all the stored information on settings.

\section{A. Dipole Power Supply}

The supply is pulse rated for $1175 \mathrm{~V}, 800$ A peak operation with a triangular wave duty cycle at a 1 second repetition rate. Initially the twelve pulse rectifier would not operate without SCR failure during inversion. It was determined that the $\mathrm{dv} / \mathrm{dt}$ transients during inversion were severe enough to fire SCRs at the wrong time resulting in these failures. A more substantial snubber circuit corrected this problem and then satisfactory operation resulted.

By having the power supply come on to full output voltage at the beginning of the rectification period the ripple at injection is minimized. The inversion level is set at approximately three quarters of the full inversion to allow 
substantial phase margin during inversion. "Light" filtering with a damped LC circuit $\left(f_{O}=100 \mathrm{~Hz}\right)$ is used to allow a fast step response at the start of rectification.

\section{B. Booster Quadrupole P.S.}

The quadrupole power supply is complicated by the high performance required to track the dipole current and the size of the supply required. To get the required dynamic performance to follow the ramp dipole current signal at injection and beyond, a current loop is closed through a series transistor bank composed of 24 Gentron MOSFETS rated 32A/400V each (EFM049-M46). A second loop is closed on the transistor-bank voltage through the $270 \mathrm{~V}, 460$ A SCR controlled power supply. A further complication of this multiloop system is that the reference voltage to the loop monitoring the transistor voltage is varied as a function of magnet current (through a look-up table in the ILC). This was necessary so that at the beginning of each cycle the transistor voltage is at a high value that then allows the initial step required to the magnet for $\mathrm{L} \mathrm{di} / \mathrm{dt}$ startup at the beginning of each cycle. Then the transistor voltage reference decreases as the current rises so that starting from an initial voltage of $100 \mathrm{~V}$ across the transistors, the voltage drops to $25 \mathrm{~V}$ by the end of rectification. So there are three loops in this system and the operation of all three had to be carefully tailored to meet the performance requirements.

An additional clamp circuit had to be added to the supply on the transistor voltage because of transients on that voltage at the top-of-ramp transition. This clamp limits the peak dissipation in the transistor bank and eliminated the early problem that was encountered of random failures among the 24 parallel transistors. The concept and initial design of the clamp circuit was provided by Henry Lancaster whose assistarice is gratefully acknowledged.

The bandwidth of the small signal transistor loop is set at $10 \mathrm{kHz}$. At this bandwidth there is a substantial difficulty with noise pick-up in the system and careful attention had to be paid to lead dress and shielding throughout the system. As the bandwidth was increased, in the check-out process, improvements were made as required and now the system is highly satisfactory and trouble free over the last year of operation. The same mode of operation for the sextupoles and correctors is employed, utilizing Techron amplifiers with satisfactory results.

\section{Line Voltage Effects}

With a rectifying-inverting system of this magnitude there must be concern for the effect of pulsing on other equipment operating from the $12 \mathrm{kV} / 480 \mathrm{~V}$ transformer that is supplying the dipole quadrupole power supplies. This includes the other pulsing supplies and low-level electronic equipment associated with the booster including the beam position monitor (BPM) which operates on low beam signal levels. The BPM system is designed ivith sufficient buffering that the pulsing of the booster dipole has not presented any problems. The booster pulsing system is fed from a $12 \mathrm{kV} / 480 \mathrm{~V}$ transforner on the pad at a 2 MVA level. This transformer is in turn fed from a 20 MVA transformer at the Grizzly Peak substation which was also used to provide power to Bevatron (a pulsed load which now has been shut down).

\section{BOOSTER TO STORAGE RING (BTS) P.S.}

The supplies needed for the booster to storage ring transport line are similar to those used in the LTB line except that the dipole supplies are large enough that it was decided to specify these supplies, and (as can be seen in table 1) the price per kilowatt turned out to be very competitive with commercially available supplies.

\section{STORAGE RING P.S. SYSTEM}

In the storage ring there are four large supplies and 220 small supplies. The large supplies are the gradient, QFA quadrupole, and SF and SD sextupole supplies. These supplies all drive families of magnets that are distributed around the storage ring in 12 sectors. The gradient power supply has the tightest specification at $+/-50$ PPM maximum allowable variation in current. These are all DC steady state supplies that normally run at currents necessary for $11 / 2 \mathrm{GeV}$ operation with the capabilities of ramping slowly up to their rated currents necessary for $1.9 \mathrm{GeV}$ operation. The other quadrupoles in the storage ring lattice are called QF and QD quadrupoles and each of the magnets have separate power supplies. These 48 quadrupole supplies are part of a family of supplies that were specified and purchased in total from Inverpower. There are five different types of supplies within this family. The two quadrupole supplies already mentioned, vertical and horizontal corrector supplies that drive separate windings on a single iron frame magnet, and supplies for the vertical/horizontal corrector windings mounted on the sextupole magnets. As can be seen from table 1, there is also a skew quad supply in this family of five different types of power supplies. These supplies range in power level from 7 $\mathrm{kW}$ to $900 \mathrm{~W}$ and all the correctors are bipolar supplies. All of these supplies are linear supplies with series pass banks of parallel FET transistors as the controlling elements. Experience in building these supplies shows that linear supplies were cost competitive with switching supplies of in the same size. The advantage of using the linears compared to switchers for approximately the same cost is the elimination of possible second-order beam effects associated with switching noise.

The gradient power supply is manufactured by PEI and is a 12 pulse steady-state rated supply. The required performance can be satisfactorily achieved with careful attention to low drift front-end design, a wide-band voltage-lcop, and a quality firing circuit designed by the manufacturer. The 12 pulse generation was specified to be achieved in this supply by using two identical octagon-primary rectifier transformers each feeding 6 pulse bridge rectifiers. This was specified because the booster twelve pulse dipole supply with delta/delta and delta/wye transformers did not provide the 12 pulse ripple that was desired but rather a combination of 6 and 12 pulse ripple. The 2 sextupole power supplies are also manufactured by PEI and although they have a lower performance requirement use the same hardware components except the water-cooled frontend package required by the gradient supply.

The QFA power supply is manufactured by Inverpower and utilizes the same hardware as their BTS power supplies with a higher performance front end. These supplies all came into operation within a minimum of difficulty and have so far proven to be quite reliable. The only reliability problem has been with the phase information transformers produced by PEI 
of which three have failed at this point. The failure is felt to be because of the very small wire that is utilized (\#35 AWG) on the $480 \mathrm{~V}$ primaries.

\section{A. Small Power Supplies}

Because of the number of these small supplies (220) that needed to be produced, an integrated approach to all of them with the same package of mechanical and electrical features was desirable. This concept was achieved through common specifications and has been carried out in an exemplary fashion by Inverpower in their design and construction. The only variation in the mechanical package is that the smaller supplies are in a 10 inch high chassis rather than the 12 inch chassis needed for the large supplies. The low powered units are fed from single phase $120 \mathrm{~V}$ power while the vertical corrector and quadrupole supplies are fed from three phase $208 \mathrm{~V}$ power. All the chassis utilize quick-disconnect water fittings and water-cooled heat sinks for the FET pass transistors.

The supplies all have the same operational layout whether large or small. Immediately behind the front panel is a printed circuit board which extends across the whole chassis. This board contains all controls, interlocks, and analog circuitry necessary for the operation of any of the power supplies. Some of the components are not used on some of the smaller supplies. Immediately in back of the master control board is the water-cooled shunt which is manufactured by VishayMann in England and has a $1 \mathrm{~V}$ max. output signal level for all units. Above the water-cooled shunt is the water-cooled heat sink for the FETs which also has thermal interlocks connected to it. The number of transistors varies greatly between the various applications. The horizontal bipolar steering supplies utilize only one FET on either side whereas the quadrupole QF supply has eight in parallel in the unipolar application. Inverpower used two different models of MOSFET, both manufactured by International Rectifier, the $150 \mathrm{~A} / 100 \mathrm{~V}$ IRFK6H150, and the 150A/200V IRFK6H250 (the bipolar supplies are quasi-complementary). In behind the water-cooled heat sink is the power transformer and in the rear is the contactor and water-flow switch.

These power supplies have proven to be reliable in operation. The biggest operational problems have been related to the water cooling circuits with flow switches failing and flow restrictors plugging. The operation of this supply family has been very satisfactory and the performance of these supplies with a $1 \mathrm{kHz}$ unity gain-bandwidth and low-thermal drift has been very adequate for all of the operations so far and anticipated.

\section{REFERENCES}

[1] L.T. Jackson and I. C. Lutz, "Magnet Power Supplies for the Advanced Light Source", Proceedings of 1989 IEEE PAC, Vol. 3, pp. 1910-1912, LBL-26012.

TABLE I - MAGNET POWER SUPPLIES

\begin{tabular}{|c|c|c|c|c|c|c|c|c|c|c|}
\hline Loc. & Type & No. & $E(V)$ & $\underline{\mathrm{I}(\mathrm{A})}$ & $\underline{P(w)}$ & $\underline{\mathrm{P}(\mathrm{kw})}$ & $\underline{\mathrm{Mfr}}$ & $\$ /$ Cost & $\$ / \mathrm{kw}$ & $+1 . \%$ \\
\hline \multirow[t]{5}{*}{ LTB } & Dipl. & 4 & 12 & 220 & & 2.6 & EMI-TCR & 3100 & 1200 & 0.65 \\
\hline & Quad & 6 & 15 & 15 & 225 & & Kepco ATE & 1600 & 7100 & 0.1 \\
\hline & Sext & 1 & 36 & 15 & 540 & & Kepco ATE & 1800 & 3300 & 0.1 \\
\hline & V Cor. & 7 & +1.40 & 6 & 240 & & Techron 7521 & 860 & 3600 & 0.1 \\
\hline & H Cor. & 7 & $+/ .40$ & 6 & 240 & & Techron 7521 & 860 & 3600 & 0.1 \\
\hline \multirow[t]{5}{*}{ Bstr. } & Dipl. & 1 & 1175 & 800 & & 940 & PEI & 57,000 & 61 & N.A. \\
\hline & Quad. & 2 & 245 & 460 & & 113 & Alpha Sci. & 40,600 & 360 & 0.03 \\
\hline & Sext. & 8 & +1.40 & 6 & 240 & & Techron 7521 & 860 & 3600 & 0.1 \\
\hline & V Cor. & 16 & +1.40 & 6 & 240 & & Techron 7521 & 860 & 3600 & 0.1 \\
\hline & H.Cor. & 16 & +1.40 & 6 & 240 & & Techron 7521 & 860 & 3600 & 0.1 \\
\hline \multirow[t]{4}{*}{ BTS } & Dipl. & 4 & 33 & 820 & & 27 & Inverpower & 12,599 & 453 & 0.1 \\
\hline & Quad. & 11 & 40 & 130 & & 5.2 & Inverpower & 5580 & 1073 & 0.05 \\
\hline & V Cor. & 9 & +1.40 & 6 & 240 & & Techron 7521 & 1186 & 5000 & 0.1 \\
\hline & H.Cor. & 9 & +1.40 & 6 & 240 & & Techron 7521 & 1186 & 5000 & 0.1 \\
\hline \multirow[t]{11}{*}{ SR } & Gradt & 1 & 535 & 1000 & & 535 & PEI & 79,400 & 148 & 0.005 \\
\hline & $\overline{\mathrm{QFA}}$ & 1 & 400 & 550 & & 220 & Inverpower & 53,000 & 241 & 0.01 \\
\hline & Sxt F & 1 & 215 & 400 & & 86 & PEI & 33,700 & 392 & 0.1 \\
\hline & Sxt D & 1 & 215 & 400 & & 86 & PEI & 33,700 & 392 & 0.1 \\
\hline & $\mathrm{QF}$ & 24 & 54 & 130 & & 7 & Inverpower & 6480 & 926 & 0.01 \\
\hline & $\mathrm{QD}$ & 24 & 40 & 130 & & 5.2 & Inverpower & 5580 & 1073 & 0.01 \\
\hline & H Cor. & 48 & $+/ .12$ & 40 & 480 & & Inverpower & 2160 & 4500 & 0.01 \\
\hline & V Cor. & 48 & $+/ .13$ & 120 & & 1.6 & Invernower & 4050 & 2531 & 0.01 \\
\hline & H CorS & 48 & $+/ .45$ & 20 & 900 & & Inverpower & 3150 & 3500 & 0.01 \\
\hline & V CorS & 24 & +1.45 & 20 & 900 & & Inverpower & 3150 & 3500 & 0.01 \\
\hline & Sk. Qd & 4 & $+/ .45$ & 20 & 900 & & Inverpower & 3150 & 3500 & 0.01 \\
\hline
\end{tabular}




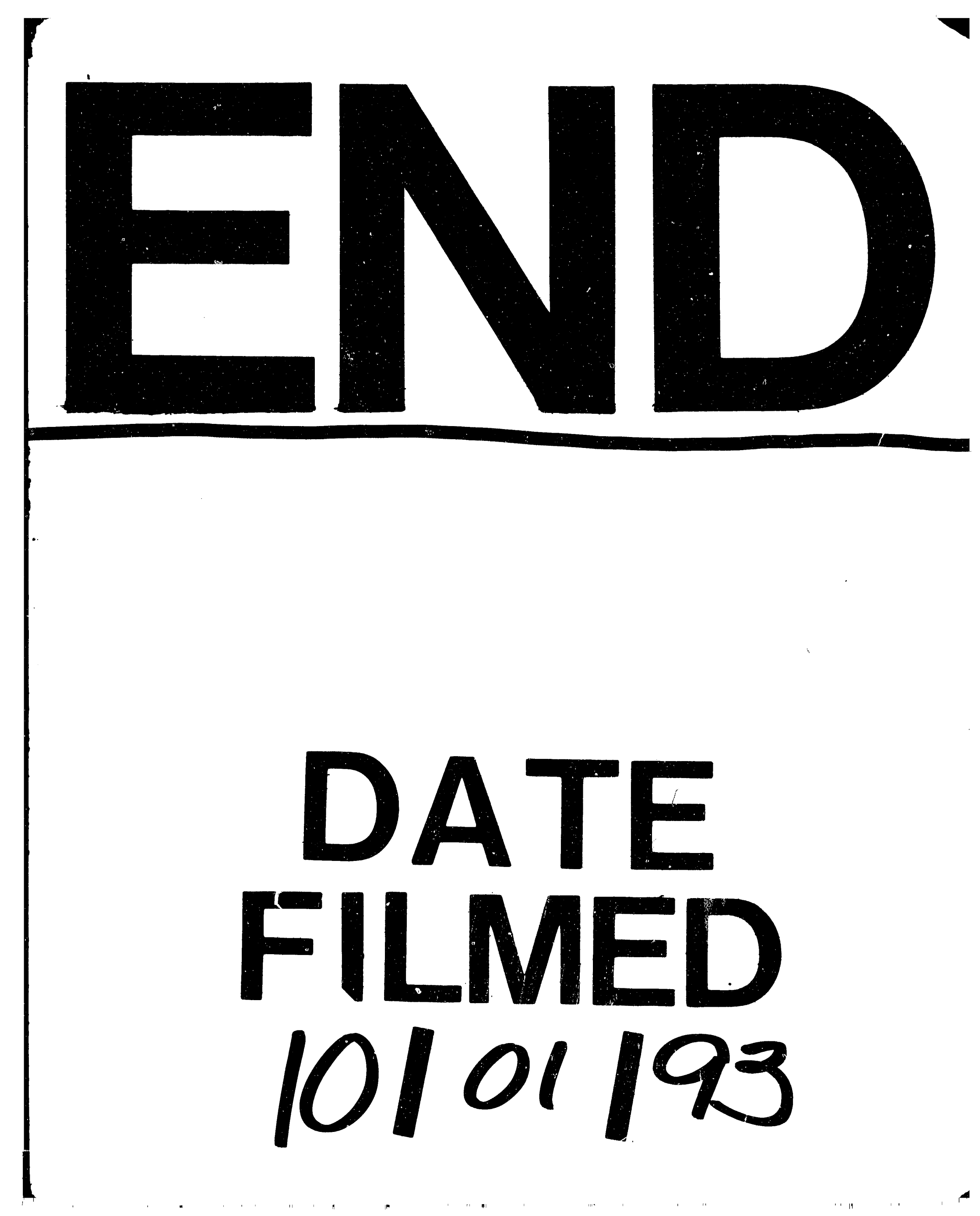




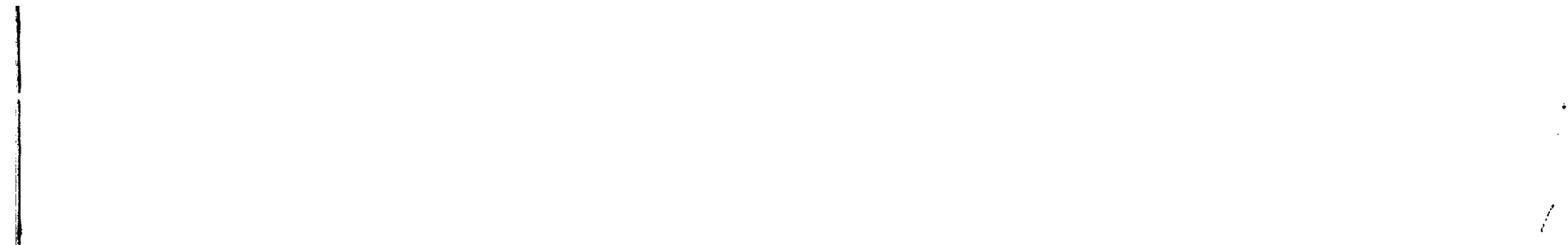

